\title{
ASSET PRICING AND WEALTH DYNAMICS-AN ADAPTIVE MODEL WITH HETEROGENEOUS AGENTS
}

\author{
CARL CHIARELLA AND XUE-ZHONG HE \\ School of Finance and Economics \\ University of Technology, Sydney \\ PO Box 123 Broadway \\ NSW 2007, Australia
}

\begin{abstract}
This paper considers a financial market with heterogeneous agents having CRRA power utility functions. To characterise asset pricing and the evolution in population and wealth dynamics, we introduce a population weighted average wealth measure. Using this measure, we develop an adaptive model of asset price and wealth dynamics. Some numerical simulations are included to illustrate the evolution of the wealth dynamics, market behaviour and market efficiency within the framework of heterogeneous agents.
\end{abstract}

Date: First Version: June 2002; Latest Draft: December 4, 2002.

Key words and phrases. Asset pricing, wealth dynamics, heterogeneity, adaptiveness. 


\section{INTRODUCTION}

The paradigm of the representative agent is built on the assumption that all agents are homogeneous with regard to their preferences, their expectations and their investment strategies. However, as already argued by Keynes in the 1930s, agents do not have sufficient knowledge of the structure of the economy to form correct mathematical expectations that would be held by all agents. Recent literature involves some departure from the classical assumptions of strict rationality and unlimited computational capacity, and introduces heterogeneity and bounded rationality of agents. In financial markets, individuals are imperfectly rational. They seek to learn about the market from their trading outcomes, as a result the market may fluctuate around the fully rational equilibrium. A number of recent models use this approach to characterize the interactions of heterogeneous agents in financial markets (e.g. Frankel and Froot (1987), Day and Huang (1990), Chiarella (1992), Lux (1995), Brock and Hommes (1997, 1998), Bullard and Duffy (1999), Farmer (1999), Farmer and Lo (1999), Franke and Nesemann (1999), Lux and Marchesi (1999) LeBaron (2000), Hommes (2001) and Chiarella and $\mathrm{He}(2002 b, 2002 c)$,).

Brock and Hommes $(1997,1998)$ propose to model economic and financial markets as an adaptive belief system (ABS), which is essentially an evolutionary competition among trading strategies. A key aspect of these models is that they exhibit expectations feedback and adaptiveness of agents. Agents adapt their beliefs over time by choosing from different predictors or expectations functions, based upon their past performance as measured by realized profits. By assuming that agents have the standard CARA utility function, the evolutionary model generates endogenous price fluctuations with similar statistical properties to those observed in financial markets. The model of Brock and Hommes has been extended in Chiarella and $\mathrm{He}(2002 c)$ by allowing agents to have different risk attitudes and different expectation formation schemes for both first and second moments of the price distribution.

The use of CARA utility functions has been standard in much of asset pricing theory. It has the characteristic of leading to demands that do not depend on the agents' wealth, but this dependence turns out to be quite crucial in developing a model exhibiting a growing price trend that is observed in the market. A CRRA utility function is sufficient to capture the interdependence of price and wealth dynamics. The selection of power utility function in the model developed here is based on a number of experimental and empirical studies, as summarized in Levy, Levy and Solomon (2000) that, "it is reasonable to assume decreasing absolute risk aversion (DARA) and constant relative risk aversion (CRRA)" (p.65). They show that the only utility function with DARA and CRRA property is the power utility function, among which, the logarithmic utility function is a special case.

Levy and Levy (1996) and Levy, Levy and Solomon (1994) consider a model where investors' optimal decisions depend on their wealth (as a result of an underlying CRRA utility function) and both price and wealth processes are intertwined and thus growing. Using numerical simulations and comparing the stock price dynamics in models with homogeneous and heterogeneous expectations, they conclude that the homogeneous expectation assumption leads to a highly inefficient market with periodic (and therefore predictable) booms and crashes while introduction of heterogeneous expectations leads to much more realistic dynamics and more efficient markets. 
Chiarella and He (2001) develop a theoretical model of interaction of portfolio decisions and wealth dynamics with heterogeneous agents having logarithmic CRRA utility function. A growth equilibrium model of both the asset price and wealth is obtained. To characterize the interaction of heterogeneous agents in financial markets and conduct a theoretical analysis, stationary models in terms of return and wealth proportions (among different types of agents) are then developed. As a special case of the general heterogeneous model, these authors consider models of homogeneous agents and of two heterogeneous agents without switching of strategies. It is found that, in these cases, the heterogeneous model can have multiple steady states and the convergence to the steady states follows an optimal selection principle - the return and wealth proportions tend to the steady state which has relatively higher return. The model developed displays the volatility clustering of the returns and the essential characteristics of the standard asset price dynamics model of continuous time finance in that the asset price is fluctuating around a geometrically growing trend.

To characterize the profitability of popular trading strategies, such as the momentum and contrarian trading strategies, we extend the model in Chiarella and He (2001) to allow agents to switch amongst different types of trading strategies and develop a simplified version of adaptive model. Under the assumption on the wealth dynamics of heterogeneous agents that, when agents switch from old strategy to new strategy, they agree to accept the average wealth level of the agents using the new strategy, the paper shows the capability to characterize some of the existing evidence on some of the anomalies observed in financial markets, including the profitability of momentum trading strategies over short time intervals and of contrarian trading strategies over long time intervals. This simplified assumption is aimed to concentrate on the profitability of particular trading strategies, not on the effect of the agents' wealth evolution on the equilibrium price. However, to characterize the equilibrium price and wealth evolution among heterogeneous agents, this assumption is rather unrealistic. The model developed in this paper is based on a more realistic assumption that, whenever a new agent switches from one group to another group, he/she brings his/her current wealth to the second group, all agents within the group agree to share their wealth. ${ }^{1}$

The key characteristics of this modelling framework are the adaptiveness, the heterogeneity and the interaction of the economic agents. The heterogeneity is expressed in terms of different views on expectations of the distribution of future returns on the risky asset. The model is developed in the discrete time setting of standard portfolio theory in that agents are allowed to revise their portfolios over each time interval, the new element being the heterogeneity of agents and the way in which they form expectations on the return distributions. Another important new element of the framework developed here is that it is able to generate an asset price evolution that has the characteristics of the geometric Brownian motion behavior observed in financial markets, viz the asset price is moving stochastically around a growing trend.

The paper is organised as follows. A general adaptive model with heterogeneous agents is developed in the following section. Some numerical examples are then included in Section 3 to illustrate the asset price, wealth dynamics.

\footnotetext{
${ }^{1}$ We would like to thank Prof. Thomas Lux to draw our attention to this crucial distinguish between these two assumptions.
} 


\section{Adaptive Model with Heterogeneous Agents}

This section is devoted to establishing an adaptive model of asset price and wealth dynamics with heterogeneous beliefs among agents. The framework of the adaptive model developed here is similar to the one in Levy and Levy (1996) and Chiarella and He (2001, 2002a). Our hypothetical financial market contains two investment choices: a stock (or index of stocks) and a bond. The bond is assumed to be a risk free asset and the stock is a risky asset. For the standard portfolio optimization problem, a model in terms of price and wealth is established first in this section. In order to characterise the evolution of agent population and wealth dynamics, a population weighted average wealth measure is introduced. Consequently the equilibrium model is reduced to a stationary model in terms of the return on the risky asset and the weighted average wealth proportions among heterogeneous investors. Based on certain performance (or fitness) measures, an adaptive mechanism is finally introduced, leading to the general adaptive model. The final model includes the dynamics of both the asset price and wealth and it characterizes three important and related issues in the study of financial market: heterogeneity, adaptiveness, and interaction of agents.

\subsection{Notation. Denote}

$p_{t}$ : Price (ex dividend) per share of the risky asset at time $t$;

$y_{t}:$ Dividend at time $t$;

$R: \quad$ Risk free return with risk free rate $r=R-1$;

$N$ : Total number of shares of the risky asset;

$H$ : Total number of investors;

$N_{i, t}$ : Number of shares acquired by agent $i$ at time $t$;

$W_{i, t}$ : Wealth of agent $i$ at time $t$;

$W_{i, 0}$ : Initial wealth of agent $i$;

$\pi_{i, t}$ : Proportion of wealth of agent $i$ invested in the risky asset at time $t$;

$\rho_{t}:$ The return on the risky asset at period $t$.

It is assumed that agents have the power utility function with different risk aversion coefficients, that is,

$$
U_{i}(W)=\frac{W^{1-\gamma_{i}}-1}{1-\gamma_{i}}, \quad \gamma_{i}>0 \quad(i=1,2, \cdots, H)
$$

Following the above notation, the return on the risky asset at period $t$ is then defined $\mathrm{by}^{2}$

$$
\rho_{t}=\frac{p_{t}-p_{t-1}+y_{t}}{p_{t-1}}
$$

\footnotetext{
${ }^{2}$ The return can also be defined by the difference of logarithms of the prices. It is known that the difference between these two definition becomes smaller as the time interval is reduced (say, from monthly to weekly or daily).
} 
2.2. Portfolio Optimization Problem of Heterogeneous Agents. Following the standard portfolio optimization approach, the wealth of agent (or investor) $i$ at time period $t+1$ is given by

$$
\begin{aligned}
W_{i, t+1} & =\left(1-\pi_{i, t}\right) W_{i, t} R+\pi_{i, t} W_{i, t}\left(1+\rho_{t+1}\right) \\
& =W_{i, t}\left[R+\pi_{i, t}\left(\rho_{t+1}-r\right)\right] .
\end{aligned}
$$

As in Brock and Hommes (1998) and Levy and Levy (1996), a Walrasian scenario is used to derive the demand equation, i.e., each trader is viewed as a price taker and the market is viewed as finding (via the Walrasian auctioneer) the price $p_{t}$ that equates the sum of these demand schedules to the supply. That is, the agents treat the period $t$ price, $p_{t}$, as parametric when solving their optimisation problem to determine $\pi_{i, t}$. Denote by $F_{t}=\left\{p_{t-1}, \cdots ; y_{t}, y_{t-1}, \cdots\right\}$ the information $\operatorname{set}^{3}$ formed at time $t$. Let $E_{t}, V_{t}$ be the conditional expectation and variance, respectively, based on $F_{t}$, and $E_{i, t}, V_{i, t}$ be the "beliefs" of investor $i$ about the conditional expectation and variance. Then it follows from (2.2) that

$$
\begin{aligned}
E_{i, t}\left(W_{i, t+1}\right) & =W_{i, t}\left[R+\pi_{i, t}\left(E_{i, t}\left(\rho_{t+1}\right)-r\right)\right], \\
V_{i, t}\left(W_{i, t+1}\right) & =W_{i, t}^{2} \pi_{i, t}^{2} V_{i, t}\left(\rho_{t+1}\right) .
\end{aligned}
$$

Consider investor $i$, who faces a given price $p_{t}$, has wealth $W_{i, t}$ and believes that the asset return is conditionally normally distributed with mean $E_{i, t}\left(\rho_{t+1}\right)$ and variance $V_{i, t}\left(\rho_{t+1}\right)$. This investor chooses a proportion $\pi_{i, t}$ of his/her wealth to be invested in the risky asset so as to maximize the expected utility of the wealth at $t+1$, as given by

$$
\max _{\pi_{i, t}} E_{i, t}\left[U\left(W_{i, t+1}\right)\right] .
$$

It follows that ${ }^{4}$ the optimum investment proportion at time $t, \pi_{i, t}$ is given by

$$
\pi_{i, t}=\frac{E_{i, t}\left(\rho_{t+1}\right)-r}{\gamma_{i} V_{i, t}\left(\rho_{t+1}\right)} .
$$

Heterogeneous beliefs are introduced via the assumption that

$$
E_{i, t}\left(\rho_{t+1}\right)=f_{i}\left(\rho_{t-1}, \cdots, \rho_{t-L_{i}}\right), \quad V_{i, t}\left(\rho_{t+1}\right)=g_{i}\left(\rho_{t-1}, \cdots, \rho_{t-L_{i}}\right)
$$

for $i=1, \cdots, H$, where $L_{i}$ are integers, $f_{i}, g_{i}$ are some deterministic functions which can differ across investors. Under this assumption, both $E_{i, t}\left(\rho_{t+1}\right)$ and $V_{i, t}\left(\rho_{t+1}\right)$ are functions of the past prices up to $t-1$, which in turn implies the optimum wealth proportion $\pi_{i, t}$, defined by (2.4), is a function of the history of the prices $\left(p_{t-1}, p_{t-2}, \cdots\right)^{5}$.

\footnotetext{
${ }^{3}$ Because of the Walrasian scenario, the hypothetical price $p_{t}$ at time $t$ is included in the information set to determine the market clearing price. However, agents form their expectations by using the past prices up to time $t-1$.

${ }^{4}$ See Appendix A.1 in Chiarella and He (2001) for details.

${ }^{5}$ In Levy and Levy (1996), the hypothetical price $p_{t}$ is included in the above conditional expectations on the return and variance. In this case, the market clearing price is solved implicitly and is much more involved mathematically. The approach adopted here is the standard one in deriving the price via the Walrasian scenario and also keeps the mathematical analysis tractable. A similar approach has been adopted in Brock and Hommes (1997), (1998) and Chiarella and He (2002c). Of course other market clearing mechanisms are possible, e.g., a market-maker. It turns out that the type of market clearing mechanism used does affect the dynamics, on this point see Chiarella and He (2002b).
} 
2.3. Market Clearing Equilibrium Price-A Growth Model. The optimum proportion of wealth invested in the risky asset, $\pi_{i, t}$, determines the number of shares at price $p_{t}$ that investor $i$ wishes to hold:

$$
N_{i, t}=\frac{\pi_{i, t} W_{i, t}}{p_{t}}
$$

Summing the demands of all agents gives the aggregate demand. The total number of shares in the market, denoted by $N$, is assumed to be fixed, and hence the market clearing equilibrium price $p_{t}$ is determined by

$$
\sum_{i=1}^{H} N_{i, t}=\sum_{i=1}^{H} \frac{\pi_{i, t} W_{i, t}}{p_{t}}=N,
$$

i.e.,

$$
\sum_{i=1}^{H} \pi_{i, t} W_{i, t}=N p_{t} .
$$

Thus, equations (2.2) and (2.6) show that, in this model, as in real markets, the equilibrium price $p_{t}$ and the wealth of investors, $W_{t} \equiv\left(W_{1, t}, \cdots, W_{H, t}\right)$, are determined simultaneously. The optimum demands of agents are functions of the price and their wealth. Also, as observed in financial markets, the model implies that both the price and the wealth are growing processes in general.

2.4. Adaptiveness and Population Distribution. We focus on a simple case where all the agents can be grouped into two types in terms of their risk aversion coefficient and conditional expectations of mean and variance of returns of the risky asset. That is, within each group, all the agents have the same risk aversion coefficient and follow the same expectation schemes on the conditional mean and variance of the return $\rho_{t+1}$, and hence the optimum wealth proportion $\left(\pi_{i, t}\right)$ invested in the risky asset for the agents are the same. Let group $j$ have $\ell_{j, t}$ agents at time $t$ with $j=1,2$. Then $\ell_{1, t}+\ell_{2, t}=H$. Denote by $n_{j, t}$ the proportion of the number of agents in group $j$, at time $t$, relative to the total number of the investors, $H$, that is,

$$
n_{j, t}=\ell_{j, t} / H, \quad \text { for } \quad j=1,2, \quad n_{1, t}+n_{2, t}=1 .
$$

Some simple examples on return and wealth dynamics when proportions of different types of agents $n_{j, t}$ are fixed over time are given in Chiarella and He (2001). However, this is a highly simplified assumption and it would be more realistic to allow agents to adjust their beliefs from time to time, based on some performance or fitness measures, as discussed later. As a consequence, the proportions of different types of agents become endogenous state variables. Therefore $\left(n_{1, t}, n_{2, t}\right)$ measures the population distribution among two different types of heterogeneous agents. The change in the distribution over time can be used to measure herd behavior among heterogeneous agents, in particular, during highly volatile periods in financial markets.

2.5. A Population Weighted Average Wealth Measure and It's Dynamics. By assuming the adaptiveness of agents' behavior, agents may switch among different groups from time to time. To track the wealth evolution of each individual agent is certainly 
an interesting and important issue, but is rather a difficult problem within the current framework. We make following simplified assumption:

(H1). Whenever a new agent switches from one group to another group, he/she brings his/her current wealth to the second group, all agents within the group agree to share their wealth.

Under this assumption, we introduce a population weighted average wealth measure to characterise and compare the wealth evolution for both groups. More precise construction of such measure is given as follows.

By the notation introduced above, the agents are distributed according to $\left(n_{1, t-1}, n_{2, t-1}\right)$ and $\left(n_{1, t}, n_{2, t}\right)$ at time $t-1$ and $t$, respectively. Note that

$$
n_{1, t}+n_{2, t}=n_{1, t-1}+n_{2, t-1}=1
$$

and

$$
\ell_{1, t}=H n_{1, t}, \quad \ell_{2, t}=H n_{2, t} .
$$

Let $\bar{W}_{j, t}$ be the average wealth of agents within group $j$ at time $t$, so that $\ell_{j, t} \bar{W}_{j, t}$ gives the total wealth of group $j$ for $j=1,2$. Following from (2.2), for group $j$, formed at time period $t-1$, the average wealth of agents at time $t$ is given by

$$
\bar{W}_{j, t}=\bar{W}_{j, t-1}\left[R+\bar{\pi}_{j, t-1}\left(\rho_{t}-r\right)\right] .
$$

Without loss of generality, we assume that $n_{1, t} \geq n_{1, t-1}$. Then $\ell_{1, t} \geq \ell_{1, t-1}$ and $\ell_{2, t} \leq \ell_{2, t-1}$. Among $\ell_{1, t}$, the number $\ell_{1, t-1}$ of agents come from the group 1 , and the rest $\ell_{1, t}-\ell_{1, t-1}$ come from the group 2 . Therefore the total wealth of group 1 at time $t, \ell_{1, t} \bar{W}_{1, t}$ is generated by $\ell_{1, t-1}$ agents from the whole group 1 and $\ell_{1, t}-\ell_{1, t-1}$ agents from group 2, formed at $t-1$. By (2.7), this total wealth of group 1 at time $t$ is given by

$$
\begin{aligned}
\ell_{1, t} \bar{W}_{1, t}= & {\left[\ell_{1, t-1}+\left(\ell_{1, t}-\ell_{1, t-1}\right)\right] \bar{W}_{1, t} } \\
= & \ell_{1, t-1} \bar{W}_{1, t-1}\left[R+\bar{\pi}_{1, t-1}\left(\rho_{t}-r\right)\right] \\
& +\left(\ell_{1, t}-\ell_{1, t-1}\right) \bar{W}_{2, t-1}\left[R+\bar{\pi}_{2, t-1}\left(\rho_{t}-r\right)\right] .
\end{aligned}
$$

For group 2, as $\ell_{2, t} \leq \ell_{2, t-1}$, the total wealth at time $t$ is generated by the total wealth of $\ell_{2, t-1}$ agents from group 2 , deducted by the total wealth of $\ell_{2, t-1}-\ell_{2, t}$ agents from group 1 , formed at time $t-1$, that is,

$$
\begin{aligned}
\ell_{2, t} \bar{W}_{1, t}= & {\left[\ell_{2, t-1}-\left(\ell_{2, t-1}-\ell_{2, t}\right)\right] \bar{W}_{1, t} } \\
= & \ell_{1, t-1} \bar{W}_{2, t-1}\left[R+\bar{\pi}_{2, t-1}\left(\rho_{t}-r\right)\right] \\
& +\left(\ell_{2, t}-\ell_{2, t-1}\right) \bar{W}_{1, t-1}\left[R+\bar{\pi}_{1, t-1}\left(\rho_{t}-r\right)\right] .
\end{aligned}
$$

Define $\bar{w}_{j, t}$ as the average wealth proportion of group $j$ weighted by population within the group at time $t$, that is,

$$
\left\{\begin{array}{l}
\bar{\omega}_{1, t}=\frac{\ell_{1, t} \bar{W}_{1, t}}{\ell_{1, t} \bar{W}_{1, t}+\ell_{2, t} \bar{W}_{2, t}}, \\
\bar{\omega}_{2, t}=\frac{\ell_{2, t} \bar{W}_{2, t}}{\ell_{1, t} \bar{W}_{1, t}+\ell_{2, t} \bar{W}_{2, t}},
\end{array}\right.
$$

We then have the following result on the evolution of the weighted wealth measure. 
Lemma 2.1. Under the assumption $\left(H_{1}\right)$, the weighted average wealth levels $\left(\tilde{w}_{1, t}, \tilde{w}_{2, t}\right)$ evolve according to

$$
\left\{\begin{array}{l}
\bar{\omega}_{1, t}=\frac{\bar{\omega}_{1, t-1}\left[R+\bar{\pi}_{1, t-1}\left(\rho_{t}-r\right)\right]+\left(1-\frac{n_{2, t}}{n_{2, t-1}}\right) \bar{\omega}_{2, t-1}\left[R+\bar{\pi}_{2, t-1}\left(\rho_{t}-r\right)\right]}{\left(2-\frac{n_{1, t}}{n_{1, t-1}}\right) \bar{\omega}_{1, t-1}\left[R+\bar{\pi}_{1, t-1}\left(\rho_{t}-r\right)\right]+\left(2-\frac{n_{2, t}}{n_{2, t-1}}\right) \bar{\omega}_{2, t-1}\left[R+\bar{\pi}_{2, t-1}\left(\rho_{t}-r\right)\right]} \\
\bar{\omega}_{2, t}=\frac{\left(1-\frac{n_{1, t}}{n_{1, t-1}}\right) \bar{\omega}_{1, t-1}\left[R+\bar{\pi}_{1, t-1}\left(\rho_{t}-r\right)\right]+\bar{\omega}_{2, t-1}\left[R+\bar{\pi}_{2, t-1}\left(\rho_{t}-r\right)\right]}{\left(2-\frac{n_{1, t}}{n_{1, t-1}}\right) \bar{\omega}_{1, t-1}\left[R+\bar{\pi}_{1, t-1}\left(\rho_{t}-r\right)\right]+\left(2-\frac{n_{2, t}}{n_{2, t-1}}\right) \bar{\omega}_{2, t-1}\left[R+\bar{\pi}_{2, t-1}\left(\rho_{t}-r\right)\right]} .
\end{array}\right.
$$

Proof. From the current notation, the total wealth $W_{t}$ at time $t$ is given by

$$
W_{t}=\ell_{1, t} \bar{W}_{1, t}+\ell_{2, t} \bar{W}_{2, t},
$$

which, by use of (2.10), can be seen to evolve according to

$$
\begin{aligned}
W_{t}= & \ell_{1, t} \bar{W}_{1, t}+\ell_{2, t} \bar{W}_{2, t} \\
= & \ell_{1, t-1} \bar{W}_{1, t-1}\left[R+\pi_{1, t-1}\left(\rho_{t}-r\right)\right]+\ell_{2, t-1} \bar{W}_{2, t-1}\left[R+\pi_{2, t-1}\left(\rho_{t}-r\right)\right] \\
& +\frac{\ell_{1, t}-\ell_{1, t-1}}{\ell_{2, t-1}} \ell_{2, t-1} \bar{W}_{2, t-1}\left[R+\bar{\pi}_{2, t-1}\left(\rho_{t}-r\right)\right] \\
& +\frac{\ell_{2, t}-\ell_{2, t-1}}{\ell_{1, t-1}} \ell_{1, t-1} \bar{W}_{1, t-1}\left[R+\bar{\pi}_{1, t-1}\left(\rho_{t}-r\right)\right] \\
= & W_{t-1}\left[\bar{\omega}_{1, t-1}\left(R+\bar{\pi}_{1, t-1}\left(\rho_{t}-r\right)\right)+\bar{\omega}_{2, t-1}\left(R+\bar{\pi}_{2, t-1}\left(\rho_{t}-r\right)\right)\right. \\
& +\left(1-\frac{\ell_{1, t}}{\ell_{1, t-1}}\right) \bar{\omega}_{1, t-1}\left(R+\bar{\pi}_{1, t-1}\left(\rho_{t}-r\right)\right) \\
& \left.+\left(1-\frac{\ell_{2, t}}{\ell_{2, t-1}}\right) \bar{\omega}_{2, t-1}\left(R+\bar{\pi}_{2, t-1}\left(\rho_{t}-r\right)\right)\right] \\
= & W_{t-1}\left[\left(2-\frac{\ell_{1, t}}{\ell_{1, t-1}}\right) \bar{\omega}_{1, t-1}\left(R+\bar{\pi}_{1, t-1}\left(\rho_{t}-r\right)\right)\right. \\
& \left.+\left(2-\frac{\ell_{2, t}}{\ell_{2, t-1}}\right) \bar{\omega}_{2, t-1}\left(R+\bar{\pi}_{2, t-1}\left(\rho_{t}-r\right)\right)\right] .
\end{aligned}
$$

Following from (2.8), (2.9), and (2.10),

$$
\begin{aligned}
\ell_{1, t} \bar{W}_{1, t}=W_{t-1}[ & \bar{\omega}_{1, t-1}\left[R+\bar{\pi}_{1, t-1}\left(\rho_{t}-r\right)\right] \\
& \left.+\left(1-\frac{\ell_{2, t}}{\ell_{2, t-1}}\right) \bar{\omega}_{2, t-1}\left[R+\bar{\pi}_{2, t-1}\left(\rho_{t}-r\right)\right]\right],
\end{aligned}
$$

and

$$
\begin{aligned}
\ell_{2, t} \bar{W}_{2, t}=W_{t-1}[ & \bar{\omega}_{2, t-1}\left(R+\bar{\pi}_{2, t-1}\left(\rho_{t}-r\right)\right) \\
& \left.+\left(1-\frac{\ell_{1, t}}{\ell_{1, t-1}}\right) \bar{\omega}_{1, t-1}\left(R+\bar{\pi}_{1, t-1}\left(\rho_{t}-r\right)\right)\right] .
\end{aligned}
$$

Then equations (2.12)-(2.14) lead to the result. 
2.6. Market Equilibrium Return. With the help of the population weighted average wealth measure, we are able to obtain the market equilibrium return $\rho_{t}$ of the risk asset.

Lemma 2.2. Under the assumption $\left(H_{1}\right)$, the market equilibrium rate of return of the risky asset satisfies

$$
\rho_{t}=r+\frac{F}{G}
$$

where

$$
\begin{aligned}
& F=\left(\alpha_{t}-R\right)\left[\bar{\omega}_{1, t-1} \bar{\pi}_{1, t-1}+\bar{\omega}_{2, t-1} \bar{\pi}_{2, t-1}\right] \\
&+R\left[\left(\bar{\pi}_{1, t}+\bar{\pi}_{2, t}\right)-\frac{n_{1, t}}{n_{1, t-1}} \bar{\pi}_{2, t} \bar{\omega}_{1, t-1}-\frac{n_{2, t}}{n_{2, t-1}} \bar{\pi}_{1, t} \bar{\omega}_{2, t-1}\right], \\
& G=\left[1-\left(\bar{\pi}_{1, t}+\bar{\pi}_{2, t}\right)\right]\left[\left(\bar{\omega}_{1, t-1} \bar{\pi}_{1, t-1}+\bar{\omega}_{2, t-1} \bar{\pi}_{2, t-1}\right)\right] \\
& \quad+\frac{n_{1, t}}{n_{1, t-1}} \bar{\omega}_{1, t-1} \bar{\pi}_{1, t-1} \bar{\pi}_{2, t}+\frac{n_{2, t}}{n_{2, t-1}} \bar{\omega}_{2, t-1} \bar{\pi}_{2, t-1} \bar{\pi}_{1, t} .
\end{aligned}
$$

and $\alpha_{t}=y_{t} / \alpha_{t}$ denotes the dividend yield.

Proof. It follows from (2.12) that

$$
\begin{aligned}
\frac{W_{t}}{W_{t-1}}= & \left(2-\frac{\ell_{1, t}}{\ell_{1, t-1}}\right) \bar{\omega}_{1, t-1}\left[R+\bar{\pi}_{1, t-1}\left(\rho_{t}-r\right)\right] \\
& +\left(2-\frac{\ell_{2, t}}{\ell_{2, t-1}}\right) \bar{\omega}_{2, t-1}\left[R+\bar{\pi}_{2, t-1}\left(\rho_{t}-r\right)\right] .
\end{aligned}
$$

The market clearing condition (2.6) implies that

$$
\ell_{1, t} \bar{\pi}_{1, t} \bar{W}_{1, t}+\ell_{2, t} \bar{\pi}_{2, t} \bar{W}_{2, t}=N p_{t}
$$

By considering (2.19) at two successive times one obtains the expression for return

$$
\frac{\ell_{1, t} \bar{\pi}_{1, t} \bar{W}_{1, t}+\ell_{2, t} \bar{\pi}_{2, t} \bar{W}_{2, t}}{\ell_{1, t-1} \bar{\pi}_{1, t-1} \bar{W}_{1, t-1}+\ell_{2, t-1} \bar{\pi}_{2, t-1} \bar{W}_{2, t-1}}=1+\rho_{t}-\alpha_{t},
$$

where $\alpha_{t}=y_{t} / p_{t-1}$ defines the dividend yield. Using the weighted average wealth measure, equation (2.20) can be written as follows:

$$
\frac{\left[\bar{\omega}_{1, t} \bar{\pi}_{1, t}+\bar{\omega}_{2, t} \bar{\pi}_{2, t}\right] W_{t}}{\left[\bar{\omega}_{1, t-1} \bar{\pi}_{1, t-1}+\bar{\omega}_{2, t-1} \bar{\pi}_{2, t-1}\right] W_{t-1}}=\left(R-\alpha_{t}\right)+\left(\rho_{t}-r\right) .
$$

Substituting (2.18) into equation (2.21),

$$
\begin{aligned}
&\left(\bar{\omega}_{1, t} \bar{\pi}_{1, t}+\bar{\omega}_{2, t} \pi_{2, t}\right) {\left[\left(2-\frac{\ell_{1, t}}{\ell_{1, t-1}}\right) \bar{\omega}_{1, t-1}\left[R+\bar{\pi}_{1, t-1}\left(\rho_{t}-r\right)\right]\right.} \\
&\left.+\left(2-\frac{\ell_{2, t}}{\ell_{2, t-1}}\right) \bar{\omega}_{2, t-1}\left[R+\bar{\pi}_{2, t-1}\left(\rho_{t}-r\right)\right]\right] \\
&=\left[\left(R-\alpha_{t}\right)+\left(\rho_{t}-r\right)\right]\left[\bar{\omega}_{1, t-1} \bar{\pi}_{1, t-1}+\bar{\omega}_{2, t-1} \bar{\pi}_{2, t-1}\right] .
\end{aligned}
$$


Substituting (2.11) into (2.22),

$$
\begin{aligned}
\left(\rho_{t}-r\right)\{[ & \left.\bar{\pi}_{1, t-1} \bar{w}_{1, t-1}+\bar{\pi}_{2, t-1} \bar{w}_{2, t-1}\right] \\
& -\bar{\pi}_{1, t}\left[\bar{\omega}_{1, t-1} \bar{\pi}_{1, t-1}++\left(1-\frac{n_{2, t}}{n_{2, t-1}}\right) \bar{\omega}_{2, t-1} \bar{\pi}_{2, t-1}\right] \\
& \left.-\bar{\pi}_{2, t}\left[\bar{\omega}_{2, t-1} \bar{\pi}_{2, t-1}++\left(1-\frac{n_{1, t}}{n_{1, t-1}}\right) \bar{\omega}_{1, t-1} \bar{\pi}_{1, t-1}\right]\right\} \\
= & R\left[\left(\bar{\pi}_{1, t}+\bar{\pi}_{2, t}\right)-\left(\bar{\pi}_{1, t-1} \bar{w}_{1, t-1}+\bar{\pi}_{2, t-1} \bar{w}_{2, t-1}\right)\right. \\
& \left.-\frac{n_{2, t}}{n_{2, t-1}} \bar{\pi}_{1, t} \bar{w}_{2, t-1}-\frac{n_{1, t}}{n_{1, t-1}} \bar{\pi}_{2, t} \bar{w}_{1, t-1}\right] \\
& +\alpha_{t}\left[\bar{\pi}_{1, t-1} \bar{w}_{1, t-1}+\bar{\pi}_{2, t-1} \bar{w}_{2, t-1}\right]
\end{aligned}
$$

Let

$$
\Gamma_{t}=\bar{\omega}_{1, t} \bar{\pi}_{1, t}+\bar{\omega}_{2, t} \bar{\pi}_{2, t}
$$

be the corresponding proportion of total weighted wealth invested in the risky asset. Then, in terms of this quantity, both equations (2.16) and (2.17) may be written as

$$
\left\{\begin{aligned}
F= & \left(\alpha_{t}-R\right) \Gamma_{t-1} \\
& +R\left[\left(\bar{\pi}_{1, t}+\bar{\pi}_{2, t}\right)-\frac{n_{1, t}}{n_{1, t-1}} \bar{\pi}_{2, t} \bar{\omega}_{1, t-1}-\frac{n_{2, t}}{n_{2, t-1}} \bar{\pi}_{1, t} \bar{\omega}_{2, t-1}\right] \\
G= & {\left[1-\left(\bar{\pi}_{1, t}+\bar{\pi}_{2, t}\right)\right] \Gamma_{t-1} } \\
& +\frac{n_{1, t}}{n_{1, t-1}} \bar{\omega}_{1, t-1} \bar{\pi}_{1, t-1} \bar{\pi}_{2, t}+\frac{n_{2, t}}{n_{2, t-1}} \bar{\omega}_{2, t-1} \bar{\pi}_{2, t-1} \bar{\pi}_{1, t} .
\end{aligned}\right.
$$

2.7. Performance Measure and Agents' Adaptiveness. Following Brock and Hommes (1997, 1998), a performance measure or fitness function, denoted $\left(\Phi_{1, t}, \Phi_{2, t}\right)$, is publicly available to all agents. Based on the performance measure agents make a (boundedly) rational choice among the predictors. This results in the Adaptive Rational Equilibrium Dynamics, introduced by Brock and Hommes (1997), an evolutionary dynamics across predictor choice which is coupled to the dynamics of the endogenous variables. In the limit as the number of agents goes to infinity, the probability that an agent $j$ chooses trading strategy $j$ is given by the well known discrete choice model or 'Gibbs' probabilities ${ }^{6}$

$$
n_{j, t}=\exp \left[\beta\left(\Phi_{j, t-1}-C_{j}\right)\right] / Z_{t} \quad Z_{t}=\sum_{j=1}^{h} \exp \left[\beta\left(\Phi_{j, t-1}-C_{j}\right)\right],
$$

where $C_{j} \geq 0$ measures the cost of the strategy $j$ for $j=1,2, \cdots, h$.

A natural performance measure or fitness function can be taken as the cumulated wealth following agents' optimal choice. More precisely,

$$
\Phi_{j, t}=\phi_{j, t}+\lambda \Phi_{j, t-1} ; \quad \phi_{j, t}=W_{0}\left[r+\left(\rho_{t}-r\right) \bar{\pi}_{j, t-1}\right]
$$

\footnotetext{
${ }^{6}$ See Manski and McFadden (1981) and Anderson, de Palma and Thisse (1993)) for extensive discussion of discrete choice models and their applications in economics.
} 
for $j=1,2$, where $W_{o}$ is the average initial wealth for both groups, $0 \leq \lambda \leq 1$ is a memory parameter measuring how strongly the past realized fitness is discounted for strategy selection, so that $\Phi_{j, t}$ may be interpreted as the accumulated average wealth of group $j$.

2.8. An Adaptive Model on Wealth Dynamics and Return. By summarising the above analysis, we obtain the following adaptive model on the evolution dynamics of weighted average wealth proportions of two types of agents and risk asset return.

Proposition 2.3. Under the assumption $\left(H_{1}\right)$, the weighted average wealth proportions of two types of agents evolve according to (2.11) and the return $\rho_{t}$ satisfies (2.23), where $\alpha_{t}$ denotes the dividend yield defined by $\alpha_{t}=y_{t} / p_{t-1}$, both function $F$ and $G$ are defined by (2.23), and the population proportions $n_{j, t}$ evolve according to (2.24), in which the fitness functions are defined by (2.25).

Equations (2.11) and (2.23) constitute a difference equation system for $\bar{w}_{j, t}$ and $\rho_{t}$ whose order depends on the choice by agents of the $L_{j}$ at equation (2.5).

2.9. A Degenerate Case-Homogeneous Model. As a special case of the adaptive model established in Proposition 2.3, assume that all the agents are homogeneous. Then

$$
\ell_{1, t}=\ell_{2, t}=H, \quad \ell_{1, t-1}=\ell_{2, t-1}=H, \quad \bar{\omega}_{1, t}=\bar{\omega}_{2, t}=\bar{\omega}_{t}
$$

and

$$
\bar{\pi}_{1, t}=\bar{\pi}_{2, t}=\bar{\pi}_{t}, \quad \gamma_{1}=\gamma_{2}=\gamma .
$$

It then follows from (2.11) and (2.23) that $\bar{w}_{t}=1$ and

$$
F_{1}=\left(\alpha_{t}-R\right) \bar{\pi}_{t-1}+R \bar{\pi}_{t}, \quad G_{1}=\bar{\pi}_{t-1}-\bar{\pi}_{t} \bar{\pi}_{t-1} .
$$

Therefore, the equilibrium return satisfies

$$
\rho_{t}=r+\frac{R\left(\bar{\pi}_{t}-\bar{\pi}_{t-1}\right)+\alpha_{t} \bar{\pi}_{t-1}}{\bar{\pi}_{t-1}\left(1-\bar{\pi}_{t}\right)}
$$

with the optimal proportion

$$
\bar{\pi}_{t}=\frac{E_{t}\left(\rho_{t+1}\right)-r}{\gamma V_{t}\left(\rho_{t+1}\right)}
$$

where $E_{t}$ and $V_{t}$ denote the common conditional mean and variance on the return of the risky asset of all agents. We will focus on the simplest case where the mean of the dividend yield is constant so that the expected fundamental rate of return is a constant, i.e.,

$$
E_{t}\left[\alpha_{t}\right]=\alpha_{o}, \quad E_{t}\left(\rho_{t+1}\right)=E_{t}\left(\rho_{t}\right)=\bar{\rho} .
$$

By assuming $V_{t}\left(\rho_{t+1}\right)=\sigma^{2}$ is a constant, the optimal proportion corresponding to the fundamental return, $\bar{\pi}$, is given by

$$
\bar{\pi}=\frac{\mathbb{E}\left(\rho_{t+1}\right)-r}{\gamma V\left(\rho_{t+1}\right)}=\frac{\bar{\rho}-r}{\gamma \sigma^{2}}
$$

and the fundamental return $\bar{\rho}$ is solved from equation

$$
(\bar{\rho}-r)(1-\bar{\pi})=\alpha_{0} \text {. }
$$


From (2.28) and (2.29)

$$
(\bar{\rho}-r)\left[1-\frac{\bar{\rho}-r}{\gamma \sigma^{2}}\right]=\alpha_{0}
$$

from which

$$
(\bar{\rho}-r)^{2}-\gamma \sigma^{2}(\bar{\rho}-r)+\gamma \sigma^{2} \alpha_{0}=0 .
$$

Thus the constant fundamental return $\bar{\rho}$ is found to be given by

$$
\begin{aligned}
\bar{\rho} & =r+\frac{1}{2}\left[\gamma \sigma^{2} \pm \sqrt{\gamma^{2} \sigma^{4}-4 \gamma \sigma^{2} \alpha_{0}}\right] \\
& =r+\frac{1}{2} \gamma \sigma^{2}\left[1 \pm \sqrt{1-\frac{4 \alpha_{0}}{\gamma \sigma^{2}}}\right]
\end{aligned}
$$

Therefore, $\bar{\rho} \in \mathbb{R}^{+}$if and only if $4 \alpha_{0} /\left(\gamma \sigma^{2}\right) \leq 1$. In particular, let $\gamma=\frac{4 \alpha_{0}}{\sigma^{2}}$, then $\bar{\rho}=r+2 \alpha_{0}$. Based on the data for the United States during the 1926-1994 period, as reported by Ibboston Associates, the annual risk-free interest rate, $r=3.7 \%$, corresponds to the average rate during that period. A dividend yield $\alpha_{o}=4.7 \%$ corresponds to the historical average yield on the S\&P500, $\bar{\rho}=r+2 \alpha_{o}=13.1 \%$ is close to the average historical rate of return of $12.2 \%$ over the period.

\section{Some ExAmples}

The adaptive model established in Proposition 2.3 is incomplete unless the conditional expectations of agents on the mean and variance of returns are specified. Different trading strategies can be incorporated into this general adaptive model as indicated by equation (2.5). It is not trivial to study nonlinear, non-stationary dynamical systems subject to random shocks. This section consider some simple examples of the adaptive model in which two simple types of agents, termed fundamentalists and trend followers, are considered in the following example. ${ }^{7}$ Neither type is fully rational in the sense used in the rational expectations literature. The information on the dividends and realised prices is publicly available to all agent types. The examples discussed below exhibits a new element of the framework developed here, that is, it is able to generate an asset price evolution that has the characteristics of the geometric Brownian motion behavior observed in financial markets, viz the asset price is moving stochastically around a growing trend.

To illustrate the time series properties generated by our adaptive model, we assume $\gamma_{1}=\gamma_{2}=\gamma=\frac{4 \alpha_{o}}{\sigma^{2}}$ and all the agents realize a long-run mean of rate return $\bar{\rho}=$ $r+2 \alpha_{o}$, which corresponds to the constant fundamental return of the homogeneous model. With two types of traders, their conditional expected returns are assumed by

$$
E_{j, t}\left(\rho_{t+1}\right)=\bar{\rho}-d_{j}\left[\bar{\rho}_{j, t-1}-\bar{\rho}\right], \quad d_{j} \in \mathbb{R}, \quad j=1,2,
$$

where

$$
\bar{\rho}_{j, t-1}=\frac{1}{L_{j}}\left[\rho_{t-1}+\rho_{t-2}+\cdots+\rho_{t-L_{j}}\right], \quad(j=1,2)
$$

with some integer $L_{j} \geq 1$. For $d_{j} \leq 0$, agents are classified as fundamentalists, believing that the moving average return over the last $L_{j}$ periods will move back to

\footnotetext{
${ }^{7}$ To simplify the analysis, we focus on the conditional mean estimation by assuming that subjective estimation of variance of all the agents' is given by a constant, that is, $V_{i, t}=\sigma^{2}$.
} 
the fundamental return with a reverting factor $d_{j}$. For $d_{j}>0$ agents are classified as trend followers, extrapolating the excess moving average return from the constant fundamental return over the last $L_{j}$ period returns.

To catch model approximation error (such as agents behaviour are driven not only by rational expected utility maximization but also by a multitude of other factors, see Levy et.al. (2000) and references cited there) and exogenous shocks to the market equilibrium, we add a noise term $\tilde{\epsilon}_{t}$ to the equilibrium rate of return equation (2.15) where $\tilde{\epsilon}_{t}$ is a random variable drawn from a normal distribution with mean of zero and volatility of $\sigma_{\epsilon}$.

Using data for the United States during the 1926-94 period, as reported by Ibbotson Associates, the annual risk-free interest rate, $r=3.7 \%$, corresponds to the average rate during that period. The history of rates of return on the stock consists of a distribution with a mean of $12.2 \%$ and a standard derivation of $20.4 \%$. A dividend yield of $\alpha_{o}=$ $4.7 \%$ corresponds to the historical average yield on the S\&P500.

In the following simulations, the time period between each trade is one day. The number of trading days in a year is selected as $K=250$ and simulations are conducted over twenty years. We choose the initial share price $p_{o}=\$ 10.00$, the annual volatility of the dividend yield $\sigma_{y}=1.5 \%$, the switching intensity $\beta=0.5$, the memory parameter $\lambda=0.6$, the initial average wealth level $W_{o}=\$ 1,000$ for all agents. The random noise term $\tilde{\epsilon}_{t}$ follows a normal distribution with mean of zero and annual volatility of $\sigma_{\epsilon}=8 \%$.

Our simulations are conducted for different combinations of $d_{1}, d_{2}, L_{1}, L_{2}$, initial average wealth $\left(\tilde{w}_{1, o}, \tilde{w}_{2, o}\right)$ and population proportions $\left(n_{1, o}, n_{2, o}\right)$, as listed in Table 1 .

\begin{tabular}{|c|c|c|c|c|c|c|}
\hline Figure & $d_{1}$ & $d_{2}$ & $L_{1}$ & $L_{2}$ & $\tilde{w}_{1, o}$ & $n_{1, o}$ \\
\hline \hline F1 (Fig.3.1) & 0 & 0.2 & & 5 & 0.5 & 0.5 \\
\hline F2 (Fig.3.2) & 0.15 & -0.1 & 3 & 5 & 0.5 & 0.5 \\
\hline F3(Fig.3.3) & 0.03 & -0.03 & 3 & 5 & {$[0.3,0.7]$} & {$[0.3,0.7]$} \\
\hline F4 (Fig.3.4) & -0.1 & 0.15 & 3 & 5 & 0.5 & 0.5 \\
\hline F5 (Fig.3.5) & -0.05 & 0.1 & 3 & 5 & {$[0.3,0.7]$} & 0.5 \\
\hline
\end{tabular}

TABLE 1. Parameters selection.

Figures 3.1-3.5 plot the time series for the rate of return of the risky asset (top left), the evolution of the average wealth of group 1 (top right), the population proportions of group 1 (bottom left), and the price of the risk asset (bottom right).

The statistical properties of those time series are conducted over the sample of 100 to 5,000, which are summarised in Table $2 .{ }^{8}$

Based on these simulations, we obtain the following observations in general:

- Comparing with the exogenous noise term (as indicated by the last row for each block of the figure in Table 2), the return series exhibits a no-normal distribution, which are characterised by either negative or positive skewness and kurtosis. The autocorrelation coefficients in Figure 3.6 are significant for

\footnotetext{
${ }^{8}$ For the chosen set of parameters, the statistical properties are optimal for different simulations
} 

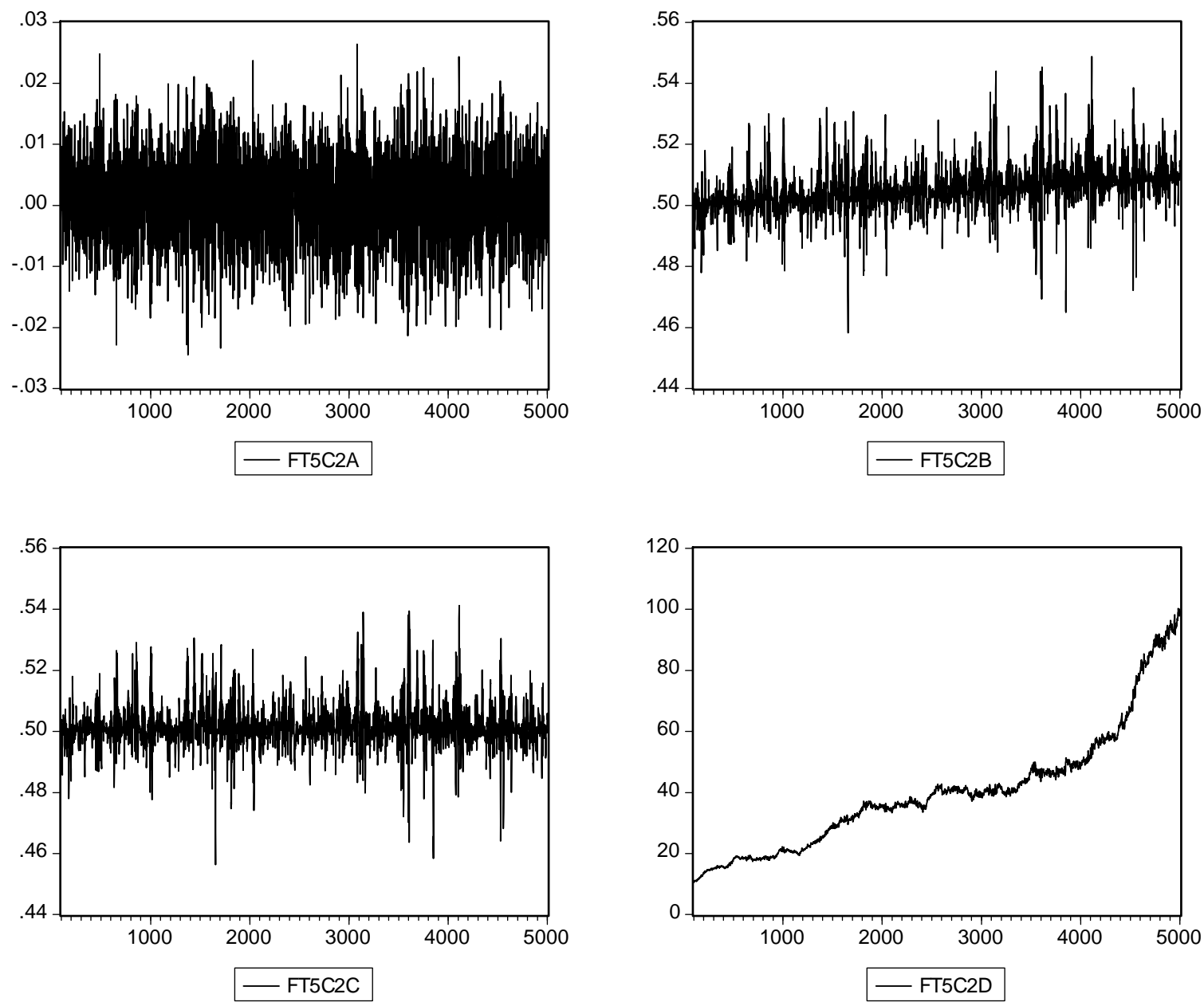

FIGURE 3.1. Time series plots for rate of return (top left), average wealth proportion (top right) and population proportion (bottom left) of the fundamentalists, and prices (bottom right) with $L_{2}=5$.

short lags. In particular, when trend followers extrapolate strongly over long windows of lag 5, as the case in Figures 3.1, the ACs in Figure 3.6(F1) have a strong oscillatory decay pattern in lags. However, such strong pattern become less persistent when the trend followers use short windows of lag 3 (as the case in Figures 3.2 and 3.6(F2)) or extrapolate weakly (as the case in Figures 3.3-3.5 and 3.6(F3-F5)).

- In terms of the average wealth among two groups, the fundamentalists tend to accumulate more wealth than the trend followers, as indicated by the time series plots (except when the reverting coefficient of the fundamentalists is zero, as in Figure 3.1). This wealth dominance of the fundamentalists is also hold even when the initial average wealth levels are not evenly distributed among the two groups (as indicated in Figures 3.3 and 3.5).

- In terms of the evolution of the population, there is no significant difference in the dominance of one group over the other, although there is strong tendency of 

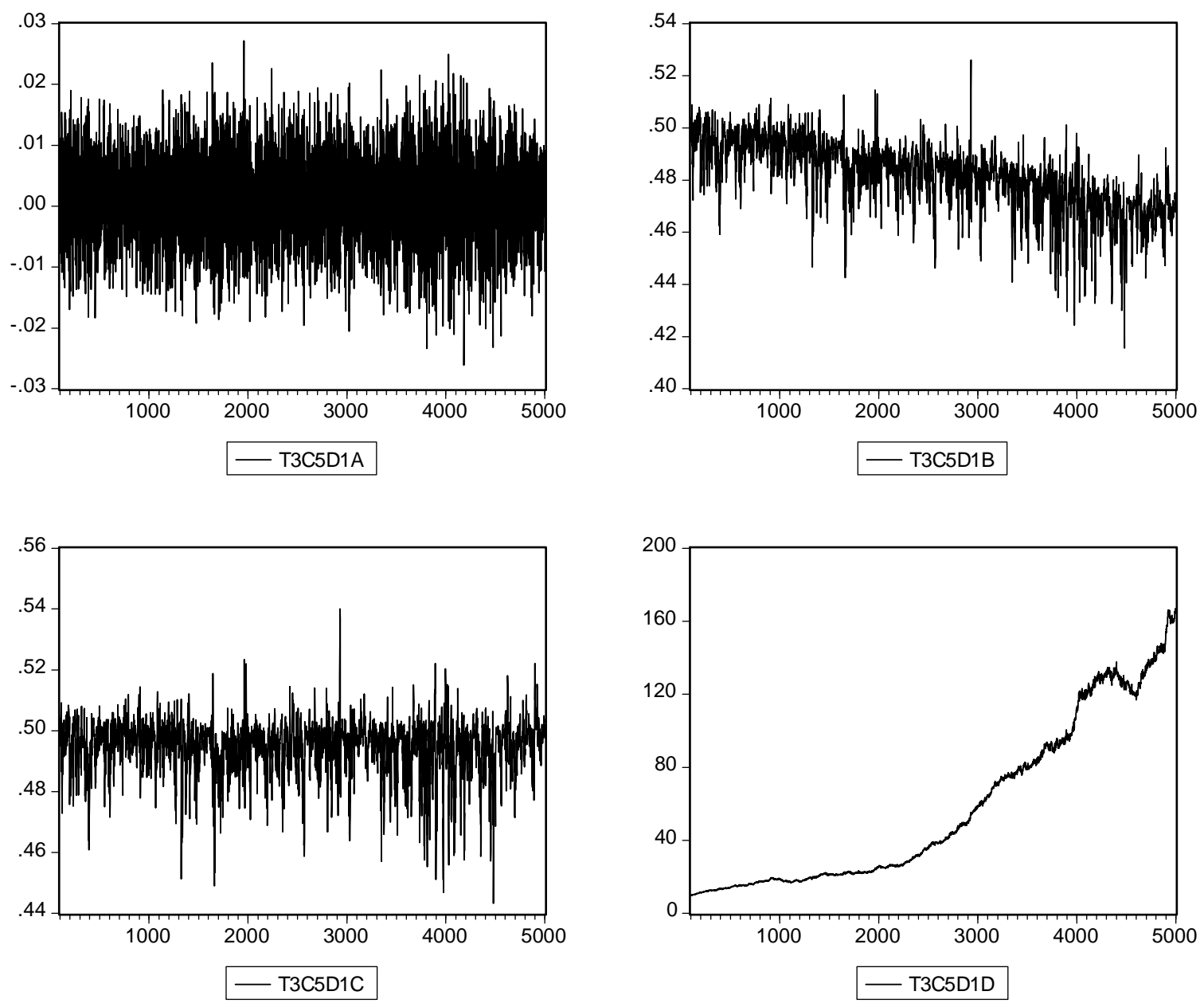

FigURE 3.2. Time series plots for rate of return (top left), average wealth proportion (top right) and population proportion (bottom left) of the trend followers, and prices (bottom right) with $L_{1}=3, L_{2}=5$ and $\tilde{w}_{1, o}=n_{1, o}=0.5$.

switching toward the fundamentalists (as indicated in Figure 3.2), even when the initial population are not evenly distributed (as the case in Figure 3.3).

- The return series tend to have higher volatility comparing with the volatility of the exogenous noise term $\tilde{\epsilon}_{t}$, as indicated in Table 2 .

- The price series moves stochastically along a growing trend, as observed for S\&P500.

- Herd behaviour can be found from the time series plots of the population proportion $n_{1, t}$, in particular, when the fundamentalists accumulate more wealth over the period. 

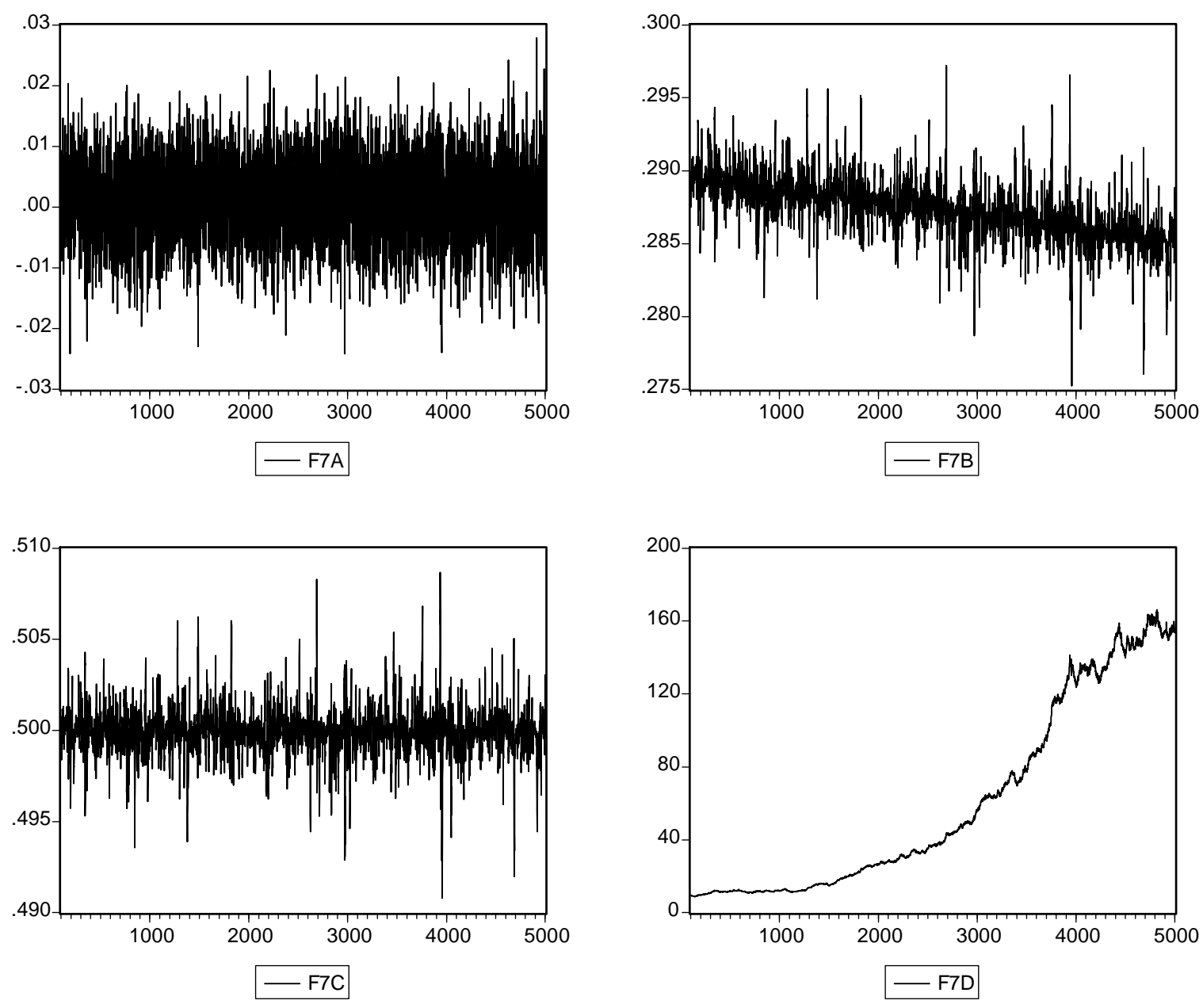

FIGURE 3.3. Time series plots for rate of return (top left), average wealth proportion (top right) and population proportion (bottom left) of the trend followers, and prices (bottom right) with $L_{1}=3, L_{2}=5$ and $\tilde{w}_{1, o}, n_{1, o} \in[0.3,0.7]$.

\section{REFERENCES}

Anderson, S. and A. de Palma and J. Thisse (1993), Discrete Choice Theory of Product Differentiation, MIT Press, Cambridge, MA.

Brock, W. and Hommes, C. (1997), 'A rational route to randomness', Econometrica 65, 1059-1095.

Brock, W. and Hommes, C. (1998), 'Heterogeneous beliefs and routes to chaos in a simple asset pricing model', Journal of Economic Dynamics and Control 22, 1235-1274.

Bullard, J. and Duffy, J. (1999), 'Using Genetic Algorithms to Model the Evolution of Heterogeneous Beliefs', Computational Economics 13, 41-60.

Chiarella, C. (1992), 'The dynamics of speculative behaviour', Annals of Operations Research 37, 101123.

Chiarella, C. and He, X. (2001), 'Asset pricing and wealth dynamics under heterogeneous expectations', Quantitative Finance 1, 509-526.

Chiarella, C. and He, X. (2002a), 'An adaptive model on asset pricing and wealth dynamics with heterogeneous trading strategies', School of Finance and Economics, University of Techonology Sydney . Working Paper No. 84. 

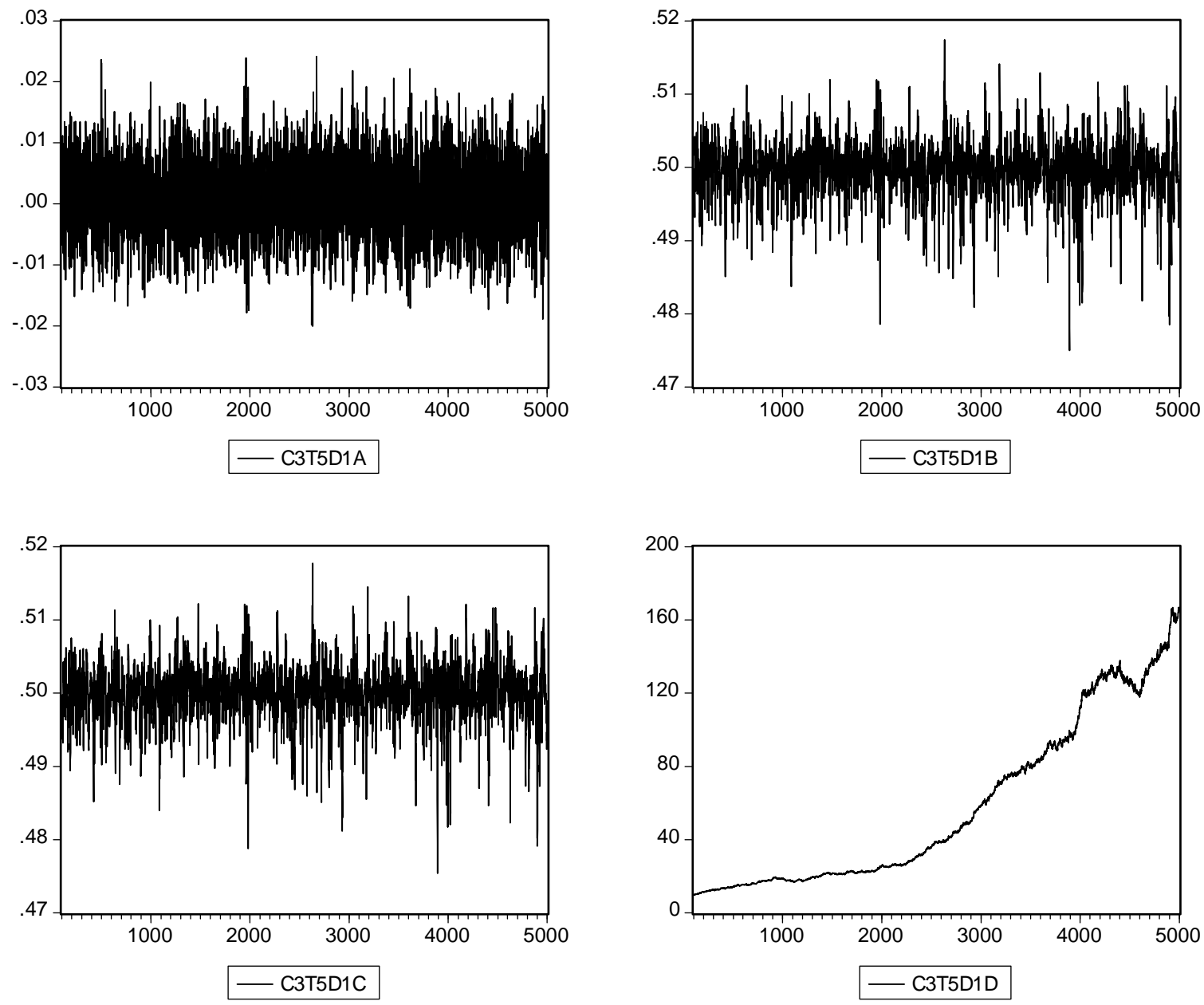

FIGURE 3.4. Time series plots for rate of return (top left), average wealth proportion (top right) and population proportion (bottom left) of the fundamentalists, and prices (bottom right) with $L_{1}=3, L_{2}=5$ and $\tilde{w}_{1, o}=n_{1, o}=0.5$.

Chiarella, C. and He, X. (2002b), 'Heterogeneous beliefs, risk and learning in a simple asset pricing model with a market maker', Macroeconomic Dynamics . in press.

Chiarella, C. and He, X. (2002c), 'Heterogeneous beliefs, risk and learning in a simple asset pricing model', Computational Economics 19, 95-132.

Day, R. and Huang, W. (1990), 'Bulls, bears and market sheep', Journal of Economic Behavior and Organization 14, 299-329.

Farmer, J. (1999), 'Physicists attempt to scale the ivory towers of finance', Computing in Science and Engineering 1, 26-39.

Farmer, J. and Lo, A. (1999), 'Frontier of finance: Evolution and efficient markets', Proceedings of the National Academy of Sciences 96, 9991-9992.

Franke, R. and Nesemann, T. (1999), 'Two destabilizing strategies may be jointly stabilizing', Journal of Economics 69, 1-18.

Frankel, F. and Froot, K. (1987), 'Using survey data to test propositions regarding exchange rate expectations', American Economic Review 77, 133-153.

Hommes, C. (2001), 'Financial markets as nonlinear adaptive evolutionary systems', Quantitative Finance 1, 149-167. 

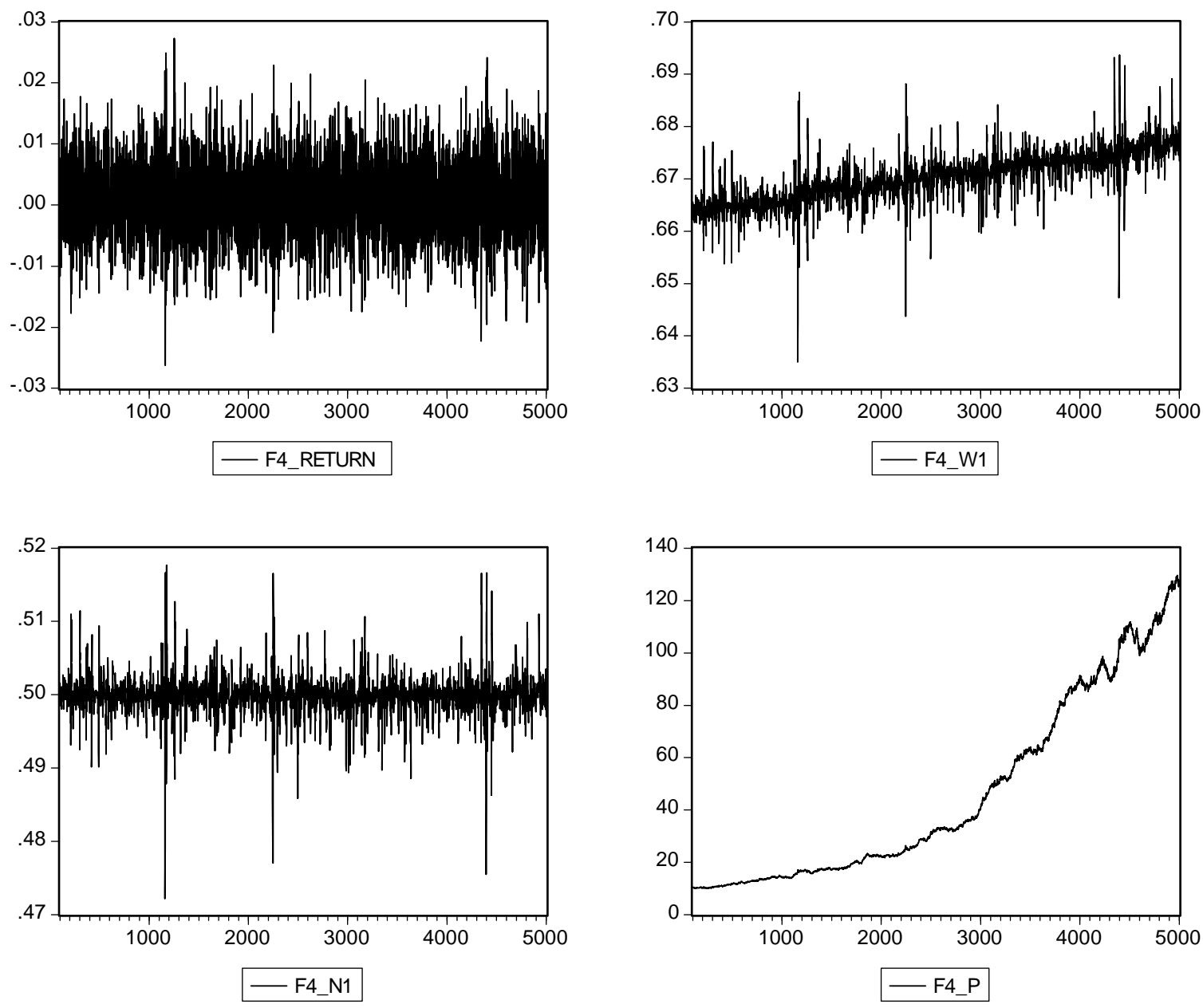

FIGURE 3.5. Time series plots for rate of return (top left), average wealth proportion (top right) and population proportion (bottom left) of the trend followers, and prices (bottom right) with $L_{1}=3, L_{2}=5$ and $\tilde{w}_{1, o} \in[0.3,0.5], n_{1, o}=0.5$.

LeBaron, B. (2000), 'Agent based computational finance: suggested readings and early research', Journal of Economic Dynamics and Control 24, 679-702.

Levy, M. and Levy, H. (1996), 'The danger of assuming homogeneous expectations', Financial Analysts Journal 52(3), 65-70.

Levy, M., Levy, H. and Solomon, S. (1994), 'A microscopic model of the stock market', Economics Letters 45, 103-111.

Levy, M., Levy, H. and Solomon, S. (2000), Microscopic Simulation of Financial Markets, from investor behavior to market phenomena, Acadmic Press, Sydney.

Lux, T. (1995), 'Herd behaviour, bubbles and crashes', Economic Journal 105, 881-896.

Lux, T. and Marchesi, M. (1999), 'Scaling and criticality in a stochastic multi-agent model of a financial markets', Nature 397(11), 498-500.

Manski, C. and McFadden, D. (1981), Structural Analysis of Discrete Data with Econometric Applications, MIT Press. 
WEALTH DYNAMICS AND ASSET PRICING WITH ADAPTIVE HETEROGENEOUS AGENTS

\begin{tabular}{|c|c|c|c|c|c|c|c|c|c|}
\hline & Mean & Median & Maximum & Minimum & Std. Dev. & Skewness & Kurtosis & Jarque-Bera & Probability \\
\hline \multicolumn{10}{|l|}{ F1 } \\
\hline$\rho_{t}$ & 0.000955 & 0.001124 & 0.026356 & -0.024443 & 0.007004 & -0.112494 & 3.097305 & \multirow[t]{3}{*}{12.27053} & \multirow[t]{4}{*}{0.002165} \\
\hline$\tilde{w}_{1, t}$ & 0.505573 & 0.505139 & 0.548637 & 0.458286 & 0.00711 & 0.343825 & 7.073934 & & \\
\hline$n_{1, t}$ & 0.501695 & 0.501134 & 0.541231 & 0.456292 & 0.006499 & 0.299697 & 9.191908 & & \\
\hline$\epsilon_{t}$ & -0.000158 & -0.000198 & 0.017939 & -0.021512 & 0.005026 & -0.02694 & 3.120077 & 3.537188 & \\
\hline \multicolumn{10}{|l|}{$\mathrm{F} 2$} \\
\hline$\rho_{t}$ & 0.001073 & 0.001195 & 0.027156 & -0.026075 & 0.00723 & -0.098077 & 2.934216 & \multirow[t]{3}{*}{8.740929} & \multirow[t]{4}{*}{0.012645} \\
\hline$\tilde{w}_{1, t}$ & 0.48156 & 0.482919 & 0.526071 & 0.415637 & 0.012387 & -0.655989 & 3.696983 & & \\
\hline$n_{1, t}$ & 0.494675 & 0.496533 & 0.540051 & 0.443308 & 0.008219 & -1.279141 & 7.067605 & & \\
\hline$\epsilon_{t}$ & $-3.29 \mathrm{E}-05$ & $4.00 \mathrm{E}-05$ & 0.020222 & -0.015843 & 0.005145 & -0.010919 & 3.03378 & 0.330403 & \\
\hline \multicolumn{10}{|l|}{ F3 } \\
\hline$\rho_{t}$ & 0.001057 & 0.001099 & 0.027838 & -0.024118 & 0.006719 & -0.077442 & 3.065987 & \multirow[t]{3}{*}{5.787952} & \multirow[t]{4}{*}{0.055356} \\
\hline$\tilde{w}_{1, t}$ & 0.287357 & 0.28739 & 0.297204 & 0.275261 & 0.001907 & -0.114369 & 4.898328 & & \\
\hline$n_{1, t}$ & 0.499938 & 0.49995 & 0.508669 & 0.490803 & 0.001209 & 0.044067 & 9.545977 & & \\
\hline$\epsilon_{t}$ & $-1.85 \mathrm{E}-05$ & $2.90 \mathrm{E}-05$ & 0.02673 & -0.024972 & 0.006368 & -0.06334 & 3.025257 & 3.407333 & \\
\hline \multicolumn{10}{|l|}{ F4 } \\
\hline$\rho_{t}$ & 0.001067 & 0.001079 & 0.024177 & -0.020027 & 0.006195 & 0.009274 & 2.967005 & \multirow[t]{3}{*}{0.292571} & \multirow[t]{4}{*}{0.863911} \\
\hline$\tilde{w}_{1, t}$ & 0.499551 & 0.499823 & 0.517439 & 0.475021 & 0.003469 & -0.948397 & 7.889587 & & \\
\hline$n_{1, t}$ & 0.499863 & 0.500127 & 0.517709 & 0.47546 & 0.003461 & -0.930515 & 7.84123 & & \\
\hline$\epsilon_{t}$ & $-3.29 \mathrm{E}-05$ & $4.00 \mathrm{E}-05$ & 0.020222 & -0.015843 & 0.005145 & -0.010919 & 3.03378 & 0.330403 & \\
\hline \multicolumn{10}{|l|}{ F5 } \\
\hline$\rho_{t}$ & 0.000988 & 0.000999 & 0.027329 & -0.026254 & 0.006091 & 0.013901 & 3.501613 & \multirow[t]{3}{*}{51.53974} & \multirow[t]{3}{*}{0} \\
\hline$\tilde{w}_{1, t}$ & 0.670145 & 0.670326 & 0.693688 & 0.635113 & 0.004716 & -0.260128 & 5.344007 & & \\
\hline$n_{1, t}$ & 0.499835 & 0.499987 & 0.517667 & 0.472231 & 0.002612 & -1.147335 & 21.29021 & & \\
\hline
\end{tabular}

TABLE 2. Statistics of time series 


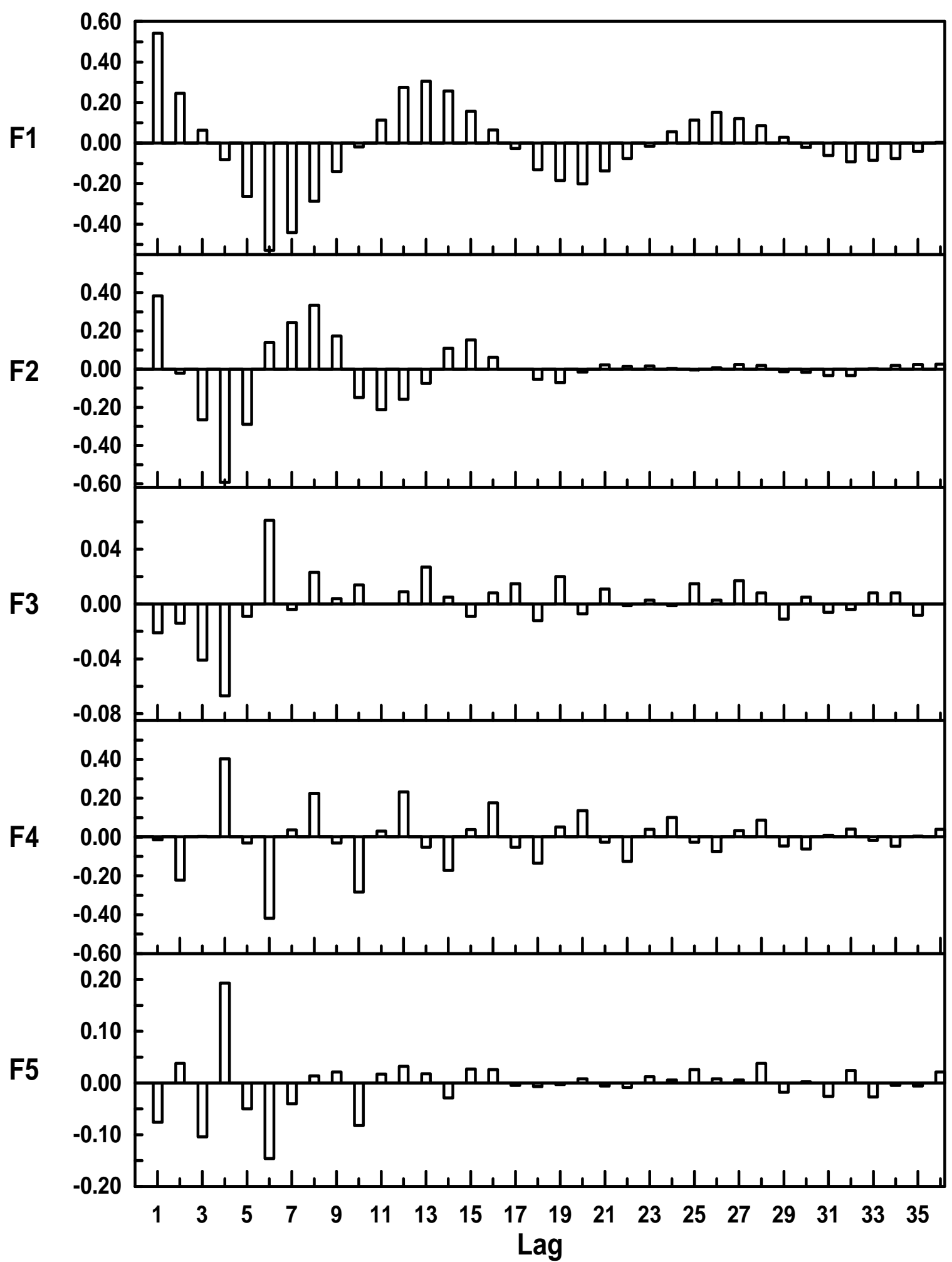

FIGURE 3.6. Autocorrelation coefficients for the rate of return series. 